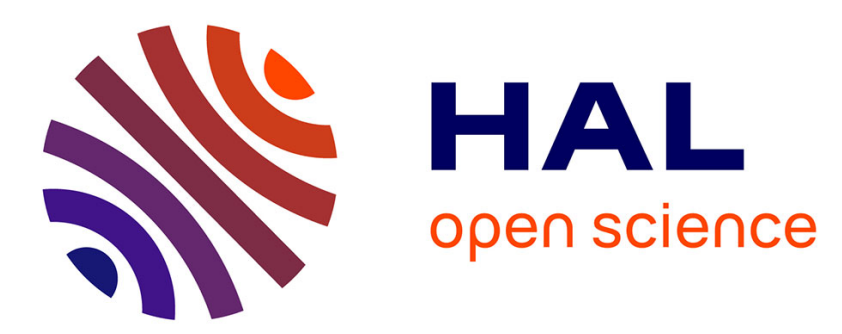

\title{
Utilisation d'un système de reconnaissance de la parole comme aide à l'acquisition orale d'une langue étrangère
}

\author{
M. T. Janot-Giorgetti, M. Lamotte
}

\section{To cite this version:}

M. T. Janot-Giorgetti, M. Lamotte. Utilisation d'un système de reconnaissance de la parole comme aide à l'acquisition orale d'une langue étrangère. Revue de Physique Appliquée, 1983, 18 (9), pp.595610. 10.1051/rphysap:01983001809059500 . jpa-00245121

\section{HAL Id: jpa-00245121 https://hal.science/jpa-00245121}

Submitted on 1 Jan 1983

HAL is a multi-disciplinary open access archive for the deposit and dissemination of scientific research documents, whether they are published or not. The documents may come from teaching and research institutions in France or abroad, or from public or private research centers.
L'archive ouverte pluridisciplinaire HAL, est destinée au dépôt et à la diffusion de documents scientifiques de niveau recherche, publiés ou non, émanant des établissements d'enseignement et de recherche français ou étrangers, des laboratoires publics ou privés. 


\title{
Utilisation d'un système de reconnaissance de la parole comme aide à l'acquisition orale d'une langue étrangère
}

\author{
M. T. Janot-Giorgetti (*) et M. Lamotte \\ Centre de Recherche en Automatique de Nancy, E.R.A. 905, \\ Université de Nancy I, B.P. nº 239, 54506 Vandœuvre-Lès-Nancy Cedex, France
}

(Reçu le ler juin 1982, révisé le 23 mars 1983, accepté le 27 mai 1983)

\begin{abstract}
Résumé. - Le système présenté ici est essentiellement une méthode de reconnaissance de la parole implantée sur un microprocesseur. Cette méthode est spécialement conçue pour l'aide à l'acquisition orale d'une langue étrangère et permet de signaler automatiquement à "l'étudiant " les fautes de prononciation, préalablement répertoriées, sur les mots d'un lexique constituant une « leçon ». Des tests effectués sur un important corpus représentatif des difficultés phonétiques et d'accent tonique de la langue anglaise, montrent les qualités et les limites du procédé.
\end{abstract}

\begin{abstract}
A computerized system of assistance in oral learning of a foreign language is presented. In this system a speech recognition method has been implemented on a microprocessor. Mispronunciations are automatically detected among those previously memorized in the system for each word of a given "lesson ". Difficulties in phonetics and position of the stressed syllables which are typical of the spoken english language have been tested to determine the effectiveness of the system.
\end{abstract}

\section{Introduction.}

Un des apports de la linguistique moderne est la mise en relief de l'importance primordiale de l'aspect oral de toute langue. Or, l'acquisition d'une prononciation correcte et d'un bon accent, ainsi que la maîtrise de l'expression orale sont souvent difficiles dans une langue étrangère. L'introduction des techniques audiovisuelles en pédagogie des langues a été une tentative dans cette voie. L'ordinateur, également, peut apporter une aide et on a vu, durant ces dix dernières années, se multiplier les projets d'Enseignement Assisté par Ordinateur, principalement aux Etats-Unis mais aussi au Japon et en Europe.

Parmi ceux qui s'adressent aux étudiants de langues, citons :

- le système PLATO développé à l'Université de l'Illinois (Etats-Unis) pour le français, l'espagnol, l'allemand, le russe, l'hébreu, le latin et même l'esperanto [1];

- le projet JACUDI, au Japon, implanté à l'Institut

(*) Nouvelle adresse : Laboratoire de la Communication Parlée, E.N.S.E.R.G., 23 avenue des Martyrs, 38031 Grenoble Cedex, France.
Kanda à Tokyo, pour l'anglais, l'allemand, le français, l'espagnol et le chinois (cité par Simon J. C. [2]);

- le projet OPE, à l'Université de Paris VII, pour l'anglais [3];

- les travaux de Nelles R. et Sennekamp M. [4] à l'Université de Fribourg, en R.F.A., sur le français.

Ces réalisations présentent un intérêt pédagogique certain, mais limité par le fait qu'elles ne s'appliquent qu'à la langue écrite. Il nous a semblé intéressant d'apporter à l'Enseignement Assisté par Ordinateur le concours de la reconnaissance automatique de la parole, offrant ainsi à l'élève un contrôle de son expression orale [5].

A notre connaissance, peu de recherches ont été faites dans cette voie. On note les travaux de Nordmann B. J. [6], à l'Université de l'Illinois, qui a proposé un projet limité à la simulation sur écran des différences de prononciation entre le professeur et l'élève, de telle sorte que ce dernier puisse se rapprocher le mieux possible du modèle proposé.

De leur côté, Kalikow D. W. et Swets I. A. [7] présentent un système de correction phonétique mis au point chez "Bolt Beranek and Newman Inc.", à Cambridge (aux Etats-Unis) pour l'apprentissage d'une langue étrangère. Il s'agit en fait, seulement d'un instructeur automatique de prononciation utilisant les capacités de mémorisation de données. 
Signalons aussi le système A.P.I. (Automated Pronunciation Instruction) développé dans le cadre du projet ARPA, initialement pour l'apprentissage du français par des espagnols, puis, dans sa seconde version, pour la rééducation des enfants mal-entendants. Le T.T. System (Teaching Training) conçu au Japon [8] est également un appareil de rééducation vocale assistée par ordinateur.

En France, des recherches récentes ont été entreprises au C.N.E.T. par Lebras J. [9] qui a mis en œuvre un algorithme rendant compte de certaines erreurs de prononciation de sujets anglophones apprenant le français (en particulier des fautes d'aspiration sur les consonnes occlusives sourdes et des fautes de diphtongaison).

On peut citer également, quoiqu'il s'agisse d'un enseignement de physique et non de langues, le processus de reconnaissance de parole continue réalisé par Bellisant C. [10] dans le cadre d'un dialogue d'enseignement assisté par ordinateur.

Le système qui fait l'objet de ce travail est baptisé MicroLEA. Ses premiers utilisateurs seront les élèves dont s'occupent les chercheurs de l'Institut de Cybernétique de Paderborn, en République Fédérale Allemande. C'est la raison pour laquelle les premiers essais [11] ont porté sur une langue normalisée (la langue internationale esperanto) que ces enseignants-chercheurs apprennent à des élèves d'une dizaine d'années dans le but de leur faciliter l'acquisition ultérieure d'une langue étrangère. MicroLEA a ensuite été testé sur d'autres langues (en particulier l'anglais, et également le français étudié par des anglophones).

Cet article présente le système MicroLEA et son application à l'apprentissage de la langue anglaise par des francophones. Les résultats concernant l'esperanto ont déjà été publiés [13].

\section{Présentation du système MicroLEA.}

Pour réaliser un appareil peu volumineux, simple et robuste, nous avons choisi, pour traiter le signal vocal, un analyseur à 8 canaux conçu au laboratoire par Vigneron C. [12] et nous avons construit un ensemble préamplificateur, accentuateur, analyseur, convertisseur analogique-numérique qui tient sur deux cartes (Fig. 1). Bien que le système ne dispose ainsi que d'informations spectrales rudimentaires, les expériences en reconnaissance de parole menées au labo- ratoire du C.R.A.N. [14, 15] ainsi que par d'autres équipes (du L.I.M.S.I. à Orsay ou du C.N.E.T. à Lannion, par exemple) ont permis de conclure à la validité d'une telle analyse [16].

Le traitement numérique est effectué par un microprocesseur qui, entouré de ses périphériques classiques, constitue une configuration informatique complète sur laquelle il a été possible d'implanter un programme conçu en fonction de l'objectif pédagogique à atteindre, c'est-à-dire permettre à l'élève de savoir si sa prononciation est correcte et, dans le cas contraire, connaitre sa faute. L'idée de base de MicroLEA est très simple : si on " apprend " à MicroLEA une liste de mots bien prononcés et également ces mêmes mots entachés des erreurs les plus courantes, il lui est ensuite possible de "reconnaître " ces fautes, grâce au programme de reconnaissance. En cas de prononciation erronée de l'élève, le système soulignera dans le libellé orthographique du mot qui apparaît sur l'écran de visualisation, soit le (ou les) phonème mal prononcé, soit une faute d'accent tonique. S'il s'agit d'une faute non prévue, il indiquera seulement que la prononciation est mauvaise. De plus, la réaudition de la voix du professeur, enregistrée sur cassette, aide l'élève à s'auto-corriger.

Les objectifs ainsi fixés impliquent que le système puisse être utilisé par un grand nombre de personnes différentes et également qu'il soit commode d'emploi aussi bien pour l'élève que pour le professeur (dans la phase "conception de la leçon "). Enfin, il paraît important, d'un point de vue didactique, de réaliser un fonctionnement en temps réel.

Sur le plan informatique, on s'est donc efforcé de remplir ces trois conditions. Si les deux dernières sont relativement faciles à satisfaire (quoique contraignantes), il n'en est pas de même pour la première car il n'est pas aisé de réaliser un système multilocuteur. En effet, toutes les expériences effectuées en reconnaissance de parole (y compris nos propres essais [17]) montrent bien que le taux de reconnaissance chute sensiblement lorsque le locuteur qui utilise l'appareil comme système de reconnaissance est différent de celui qui a réalisé l'apprentissage. La solution que nous proposons ne résoud pas complétement ce problème mais les résultats obtenus montrent qu'elle en constitue une assez bonne approche. En effet, les performances du système se trouvent nettement améliorées lorsque l'apprentissage est effectué avec plusieurs locuteurs

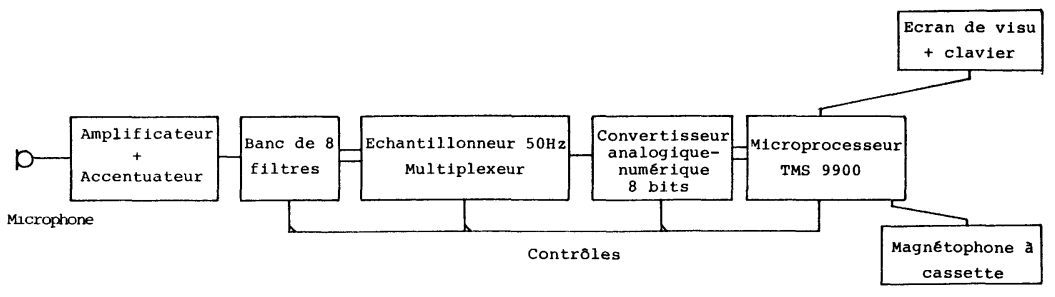

Fig. 1. - Schéma d'ensemble du matériel inclus dans le système MicroLEA.

[General description of the MicroLEA hardware.] 
de sexe, d'âge et d'origine régionale différents [18]. De plus, le système est conçu de telle sorte que la comparaison se fasse chaque fois entre le mot prononcé et un très petit nombre de mots, ceci quel que soit le volume total de la leçon. Or, on sait bien que le taux de reconnaissance décroît lorsque la taille du lexique s'accroît. On a donc évité cet écueil.

L'utilisation d'une technique de reconnaissance dérivée de la programmation dynamique s'est révélée satisfaisante car elle permet de s'affranchir des différences de durée pour un même mot prononcé par deux personnes différentes (ici le professeur et l'élève). Cela n'est pas original en soi puisque cette méthode a déjà été utilisée très largement $[19,20]$ depuis les travaux de Sakoe et Chiba au Japon [21], suite à ceux de Vintsiuk en U.R.S.S. [22]. L'intérêt du travail présenté ici réside plutôt dans l'application qui en est tentée en enseignement assisté par ordinateur.

Pour des raisons de rapidité de fonctionnement (objectif : le temps réel) et de simplicité des calculs, on utilise en fait une méthode sous-optimale de recherche de la bonne solution qui est une simplification du véritable algorithme de programmation dynamique. On l'appelle généralement « comparaison dynamique à optimum local » ou plus brièvement " comparaison dynamique ". On trouvera en annexe un exposé rapide du principe de la méthode et de son application à la reconnaissance de la parole.

S'il est souvent difficile d'évaluer les performances réelles d'un processus de reconnaissance car il y a adaptation du locuteur à la machine, ici au contraire, c'est précisément l'effet recherché puisque l'élève doit s'efforcer d'imiter un des modèles qui lui sont donnés.

\section{Conception et déroulement d'une leçon.}

MicroLEA est constitué d'une mosaïque de sousprogrammes gérés par un "programme principal» qui permet le dialogue avec les utilisateurs, à savoir le professeur dans la phase de conception de l'enseignement programmé, et l'élève au cours de la leçon proprement dite. Le dialogue machine-opérateur (professeur ou élève) se fait classiquement par l'intermédiaire d'un clavier, d'un écran de visualisation et d'un microphone.

C'est grâce au programme principal que l'opérateur peut faire exécuter le sous-programme correspondant à la commande sélectionnée. L'ensemble de ces commandes apparaît sur l'organigramme de la figure 2 , et l'organigramme de la figure 3 montre comment se déroule la phase de conception de la leçon par le professeur.

3.1 LE ROLE DU PROFESSEUR. - Le professeur devra composer la leçon et pour cela :

1) Choisir les mots à étudier en fonction des difficultés de prononciation adaptées au niveau de l'élève.

2) Réaliser l'apprentissage, donc entrer : (voir Fig. 2)

- soit la lettre $\AA$ lorsqu'il commence cette phase de son travail;

- soit la lettre $\mathrm{C}$ lorsqu'il décide de rajouter des mots à une leçon déjà enregistrée. Le sous-programme d'acquisition permet de ranger toutes les informations numériques concernant le mot prononcé, ainsi que le libellé orthographique.

3) S'assurer que tous les mots dits par lui ont été correctement acquis par le système. En entrant $\mathbb{R}$, il fait appel au sous-programme de reconnaissance. Si le système ne reconnaît pas un de ces mots lorsqu'il le prononce de nouveau, il convient de l'effacer en appelant le sous-programme d'effacement à l'aide de la commande $\mathrm{E}$ et le professeur peut refaire l'apprentissage de ce mot grâce à la commande $\mathrm{C}$.

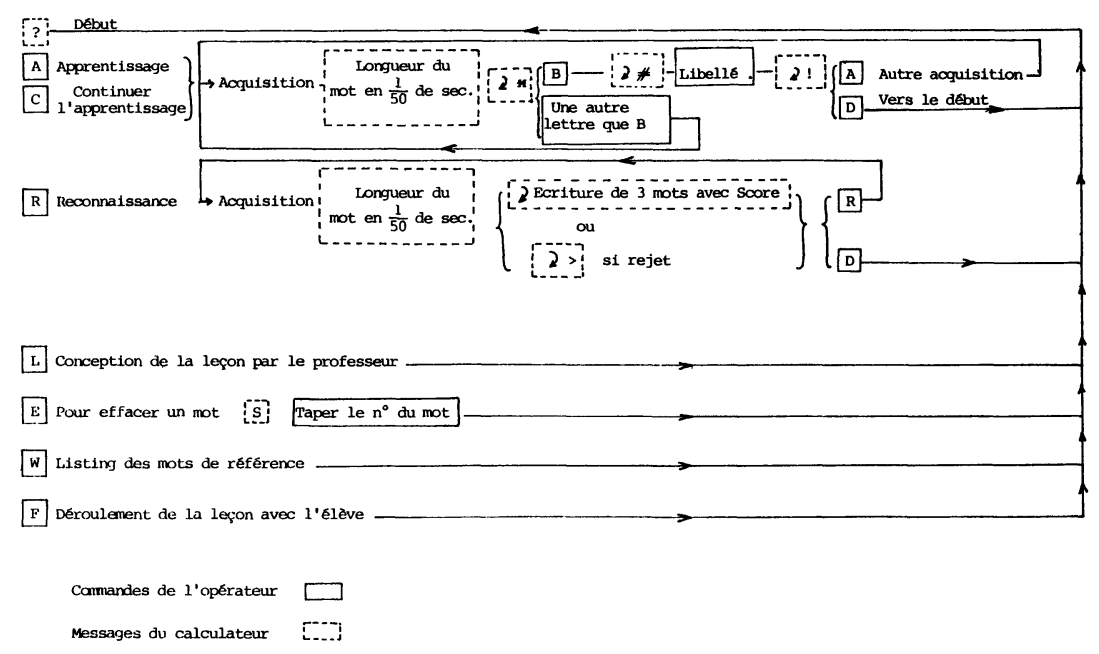

Fig. 2. - Descriptif général des sous-programmes gérés par le programme principal de MicroLEA.

[Main characteristics of the subroutines in the MicroLEA software.] 
4) Constituer un "répertoire" des mots de la leçon, indiquant, pour chaque mot, le nombre de versions (bonnes et erronées) enregistrées et les numéros repérant ces mots (commande $\mathrm{L}$ ).

5) Procéder à l'enregistrement sur cassette du " répertoire " et de l'ensemble des données de référence mémorisées pendant la phase d'apprentissage.

6) Définir la durée $Z_{\mathrm{x}}$ du déroulement de la cassette pour passer d'un mot au suivant.

7) Choisir le nombre de répétitions $V_{\mathrm{n}}$ offertes à l'élève dans le cas de mauvaise prononciation. De plus, s'il estime qu'une bonne prononciation n'est acquise avec certitude et de façon durable qu'après plusieurs essais, il peut choisir le nombre de répétitions $W_{\mathrm{n}}$ offertes à l'élève dans le cas d'une bonne prononciation.

8) Procéder à l'écriture sur cassette de ces paramètres.

9) Enregistrer sa voix sur la même cassette pendant le temps $Z_{\mathbf{x}}$.

10) Demander éventuellement un listing des mots de référence (commande $\mathrm{W}$ ).

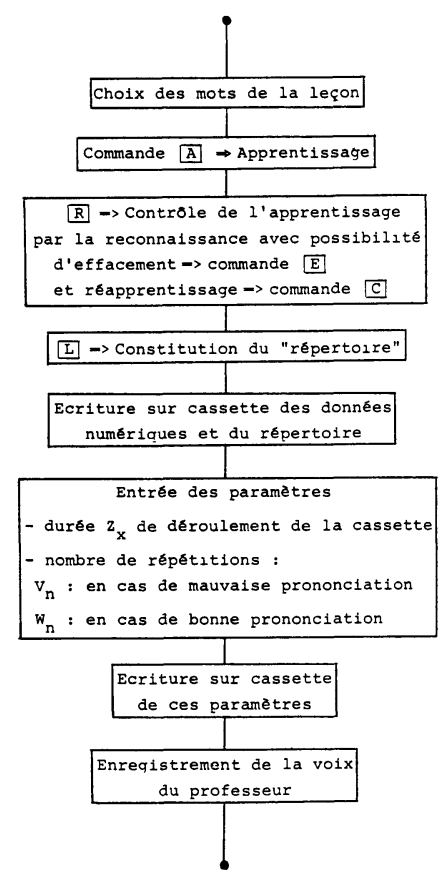

Fig. 3. - Schéma descriptif des opérations à effectuer par le professeur lors de la conception d'une leçon.

[Sketchy description of what to do for the recording of one lesson in MicroLEA memories.

Cette phase de conception étant achevée, il restera, le jour de la leçon, à charger les mémoires de MicroLEA en procédant à la lecture des données numériques (commande $[\mathrm{F}$ ). C'est l'élève qui le fait lorsque le système le lui demande au début de la leçon.

3.2 Le TRAVAIL DE L'ÉLÈVE. - Quant à l'élève, j travaille de la façon suivante [Fig. 4] : après avoir

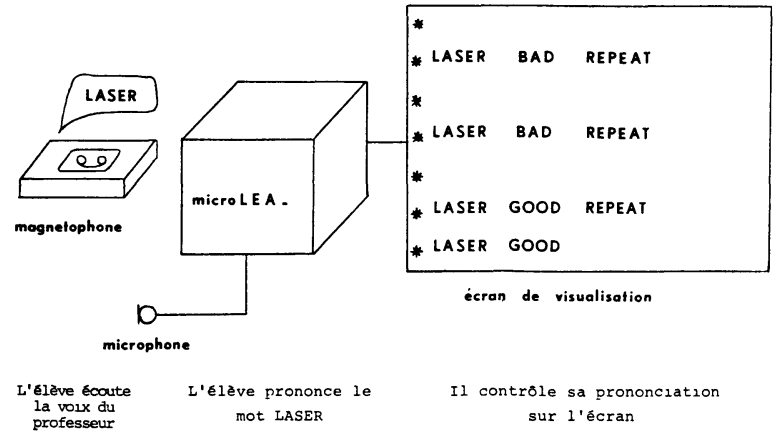

Fig. 4. - Illustration du déroulement d'une leçon pour un élève utilisant MicroLEA.

[Pictural representation of the use of MicroLEA by a " student ".

entendu le mot "Laser", par exemple, il le dit à son tour dans un microphone, puis regarde sur la console de visualisation quel est le message délivré par MicroLEA.

* Si sa prononciation a été incorrecte, il verra BAD suivi de REPEAT et le libellé du mot souligné à l'endroit de la faute de prononciation.

$$
\begin{gathered}
\text { Par exemple LASER s'il a dit /lezə/ } \\
\text { au lieu de /leizə/ } \\
\text { ou bien LASER s'il a placé l'accent tonique } \\
\text { sur la dernière syllabe au lieu } \\
\text { de la première. }
\end{gathered}
$$

* Si le mot prononcé s'éloigne trop du modèle proposé par le professeur, en particulier s'il présente une faute non prévue par l'apprentissage, il n'y aura que le message BAD REPEAT sans autre indication.

* Si sa prononciation est correcte, il lira GOOD.

Dans les deux premiers cas, il entendra automatiquement la voix de son professeur répéter le mot étudié.

Dans le dernier cas, il peut être invité :

- soit à passer directement à l'audition du mot suivant et à la suite du travail ;

- soit à répéter le mot immédiatement (et ceci $W_{\mathrm{n}}$ fois) si le professeur le désire.

Il lira alors le message GOOD suivi de REPEAT tant qu'il lui sera demandé de répéter et seulement le message GOOD lorsque le moment sera venu de passer au mot suivant.

Toutes ces possibilités font de MicroLEA un appareil très simple qui permet d'instaurer un vrai dialogue avec l'élève, même en l'absence du professeur.

\section{Le processus de reconnaissance : Résultats.}

Cette partie de MicroLEA correspondant au processus de reconnaissance, a été testée séparément pour avoir des informations quantitatives sur sa validité et, en particulier, sur son aptitude à séparer deux mots phonétiquement très voisins. Des essais systématiques portant sur un vaste corpus en anglais (de l'ordre de 
300 mots) ont été effectués avec l'aide des enseignantschercheurs du Centre de Pédagogie et d'Applications Pédagogiques en Langues de Nancy.

Les difficultés de prononciation que l'anglais présente pour un français sont sur trois niveaux :

- au niveau de certains sons correspondant à des positions articulatoires malaisées pour un Français. Les bases articulatoires du français et de l'anglais sont en effet très différentes, parfois complètement opposées;

- au niveau du mot car la mise en relief de certaines unités plus grandes que les phonèmes (diphones ou syllabes, par exemple) ne suit pas de règles bien établies. En anglais, la place de l'accent tonique varie d'un mot à l'autre et, de plus, c'est un phénomène phonétique porteur de signification. Il joue donc un rôle linguistique important qu'il importe de bien connaître (c'est ainsi que le mot "export " est un substantif qui signifie " exportation ", si l'accent expiratoire porte sur la première syllabe, ou un verbe signifiant « exporter » si on met l'accent sur la deuxième syllabe [23]);

- au niveau de la phrase car, en plus de « l'accent de mot » (suivant la terminologie traditionnelle [24]), il existe un rythme et une intonation propres à l'anglais et difficiles à acquérir pour un francophone.

Seuls les deux premiers points seront abordés ici puisque le système ne traite pour le moment que des mots isolés.

Les listes de mots-tests utilisés sont extraites d'un manuel de phonétique et de phonologie anglaise [25], rédigé à l'intention de francophones. Le tableau I illustre, par des exemples de mots, les oppositions consonantiques fondamentales de l'anglais, le tableau II les oppositions vocaliques et le tableau III

Tableau I. - Les oppositions consonantiques fondamentales de l'anglais.

[Basic consonantal oppositions in English.]

\begin{tabular}{|c|c|c|c|c|c|c|}
\hline Groupe $n^{\circ} 1$ & $\begin{array}{l}b^{\prime} \\
b\end{array}$ & $\begin{array}{l}\neq p \\
\neq m\end{array}$ & $\begin{array}{l}\text { bin } \\
\text { boor }\end{array}$ & $\begin{array}{l}\text { pin } \\
\text { moor }\end{array}$ & $\begin{array}{l}\text { cub } \\
\text { rib }\end{array}$ & $\begin{array}{l}\text { cup } \\
\text { rim }\end{array}$ \\
\hline Groupe $n^{\circ} 2$ & $\begin{array}{l}d \\
d\end{array}$ & $\begin{array}{l}=t \\
=n\end{array}$ & $\begin{array}{l}\text { down } \\
\text { dame }\end{array}$ & $\begin{array}{l}\text { town } \\
\text { name }\end{array}$ & $\begin{array}{l}\text { bid } \\
\text { bead }\end{array}$ & $\begin{array}{l}\text { bit } \\
\text { bean }\end{array}$ \\
\hline Groupe $n^{\circ} 3$ & $\begin{array}{l}n \\
g\end{array}$ & $\begin{array}{l}=n \\
=k\end{array}$ & $\begin{array}{l}\text { ton } \\
\text { goat }\end{array}$ & $\begin{array}{l}\text { tongue } \\
\text { coat }\end{array}$ & $\begin{array}{l}\text { sin } \\
\text { bag }\end{array}$ & $\begin{array}{l}\text { sing } \\
\text { back }\end{array}$ \\
\hline Groupe $n^{\circ} 4$ & & $\begin{array}{l}\neq f \\
\neq s\end{array}$ & $\begin{array}{l}\text { van } \\
\text { rise }\end{array}$ & $\begin{array}{l}\text { fan } \\
\text { rice }\end{array}$ & $\begin{array}{l}\text { leave } \\
\text { seize }\end{array}$ & $\begin{array}{l}\text { leaf } \\
\text { cease }\end{array}$ \\
\hline Groupe $n^{\circ} 5$ & & $\begin{array}{l}\neq \partial \\
\neq \theta\end{array}$ & $\begin{array}{l}\text { breeze } \\
\text { sin }\end{array}$ & $\begin{array}{l}\text { breathe } \\
\text { thin }\end{array}$ & $\begin{array}{l}\text { sees } \\
\text { force }\end{array}$ & $\begin{array}{l}\text { seethe } \\
\text { fourth }\end{array}$ \\
\hline Groupe $n^{\circ} 6$ & $\int_{d s}$ & $\begin{array}{l}\neq \mathrm{t} s \\
\neq \mathrm{t} s\end{array}$ & $\begin{array}{l}\text { ship } \\
\text { Jane }\end{array}$ & $\begin{array}{l}\text { chip } \\
\text { chain }\end{array}$ & $\begin{array}{l}\text { wish } \\
\text { age }\end{array}$ & $\begin{array}{l}\text { witch } \\
\text { "h" }\end{array}$ \\
\hline Groupe $n^{\circ} 7$ & $\begin{array}{l}\text { ts } \\
\text { ds }\end{array}$ & $\begin{array}{l}\neq \operatorname{tr} \\
\neq \text { tr }\end{array}$ & $\begin{array}{l}\text { chain } \\
\text { Jane }\end{array}$ & $\begin{array}{l}\text { train } \\
\text { train }\end{array}$ & $\begin{array}{l}\text { chick } \\
\text { Jim }\end{array}$ & $\begin{array}{l}\text { trick } \\
\text { trim }\end{array}$ \\
\hline Groupe $n^{\circ} 8$ & $\begin{array}{l}d^{3} \\
h\end{array}$ & $\begin{array}{l}\neq d r \\
\neq \phi(=0)\end{array}$ & $\begin{array}{l}\text { Jane } \\
\text { hair }\end{array}$ & $\begin{array}{l}\text { drain } \\
\text { air }\end{array}$ & $\begin{array}{l}\text { jam } \\
\text { hedge }\end{array}$ & $\begin{array}{l}\text { dram } \\
\text { edge }\end{array}$ \\
\hline
\end{tabular}

Tableau II. - Les oppositions vocaliques fondamentales de l'anglais.

[Basic vocalic oppositions in English.]

\begin{tabular}{|c|c|c|c|c|c|c|}
\hline Groupe $n^{\circ} 1$ & $\begin{array}{l}i: \\
i:\end{array}$ & 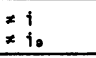 & $\begin{array}{l}\text { leave } \\
\text { bee }\end{array}$ & $\begin{array}{l}\text { live } \\
\text { beer }\end{array}$ & $\begin{array}{l}\text { sheep } \\
\text { fee }\end{array}$ & $\begin{array}{l}\text { ship } \\
\text { fear }\end{array}$ \\
\hline Groupe $n^{\circ} 2$ & $i_{0}$ & $\begin{array}{l}\neq \varepsilon \theta \\
\neq \mathrm{e}\end{array}$ & $\begin{array}{l}\text { beer } \\
\text { pin }\end{array}$ & $\begin{array}{l}\text { bear } \\
\text { pen }\end{array}$ & $\begin{array}{l}\text { cheers } \\
\text { bitter }\end{array}$ & $\begin{array}{l}\text { chairs } \\
\text { better }\end{array}$ \\
\hline Groupe $n^{\circ} 3$ & $\begin{array}{l}e \\
\text { ei }\end{array}$ & $\begin{array}{l}\mathrm{ei} \\
\times \mathrm{ai}\end{array}$ & $\begin{array}{l}\text { pen } \\
\text { pane }\end{array}$ & $\begin{array}{l}\text { pane } \\
\text { pine }\end{array}$ & $\begin{array}{l}\text { pepper } \\
\text { fate }\end{array}$ & $\begin{array}{l}\text { paper } \\
\text { fight }\end{array}$ \\
\hline Groupe $n^{\circ} 4$ & $\begin{array}{l}e \\
*\end{array}$ & $\neq=$ & $\begin{array}{l}\text { pen } \\
\text { ham }\end{array}$ & $\begin{array}{l}\text { pan } \\
\text { harm }\end{array}$ & $\begin{array}{l}\text { mess } \\
\text { hat }\end{array}$ & $\begin{array}{l}\text { mass } \\
\text { heart }\end{array}$ \\
\hline Groupe $n^{\circ} 5$ & a: & $\neq 0$ & $\begin{array}{l}\text { guard } \\
\text { card }\end{array}$ & $\begin{array}{l}\text { god } \\
\text { cod }\end{array}$ & cart & $\cot$ \\
\hline Groupe $n^{\circ} 6$ & , & $x_{0}:$ & $\begin{array}{l}\text { cod } \\
\text { sod }\end{array}$ & $\begin{array}{l}\text { cord } \\
\text { sword }\end{array}$ & $\begin{array}{l}\text { cot } \\
\text { pot }\end{array}$ & $\begin{array}{l}\text { court } \\
\text { port }\end{array}$ \\
\hline Groupe $n^{\circ} 7$ & 0 & $\neq$ ou $(\cdot u)$ & $\begin{array}{l}\text { cod } \\
\text { rod }\end{array}$ & $\begin{array}{l}\text { code } \\
\text { road }\end{array}$ & $\begin{array}{l}\text { cot } \\
\text { cock }\end{array}$ & $\begin{array}{l}\text { coat } \\
\text { coke }\end{array}$ \\
\hline Groupe $n^{\circ} 8$ & $\begin{array}{l}0: \\
\text { ou }(\circ u)\end{array}$ & $\begin{array}{l}\neq \text { ou }(\bullet u) \\
\neq a u\end{array}$ & $\begin{array}{l}\text { ball } \\
\text { tone }\end{array}$ & $\begin{array}{l}\text { bowl } \\
\text { town }\end{array}$ & $\begin{array}{l}\text { cork } \\
\text { boat }\end{array}$ & $\begin{array}{l}\text { coke } \\
\text { bout }\end{array}$ \\
\hline Groupe $n^{\circ} 9$ & $\begin{array}{l}u \\
u\end{array}$ & $\begin{array}{l}\neq \mathrm{u}: \\
\ldots 0\end{array}$ & $\begin{array}{l}\text { full } \\
\text { good }\end{array}$ & $\begin{array}{l}\text { fool } \\
\text { god }\end{array}$ & $\begin{array}{l}\text { pull } \\
\text { could }\end{array}$ & $\begin{array}{l}\text { pool } \\
\text { cod }\end{array}$ \\
\hline Groupe $n^{\circ} 10$ & $\begin{array}{l}\text { u } \\
\text { u: }\end{array}$ & $\begin{array}{l}\neq \text { ou }(\circ u) \\
\neq \text { ou }(\bullet u)\end{array}$ & $\begin{array}{l}\text { full } \\
\text { fool }\end{array}$ & $\begin{array}{l}\text { fool } \\
\text { foal }\end{array}$ & $\begin{array}{l}\text { pul1 } \\
\text { pool }\end{array}$ & $\begin{array}{l}\text { pool } \\
\text { pole }\end{array}$ \\
\hline Groupe $n^{\circ} \quad 11$ & $\begin{array}{l}\text { u: } \\
\text { u: }\end{array}$ & $\begin{array}{l}\neq \text { ou(bu) } \\
\neq \text { wo } \\
\text { wo }\end{array}$ & $\begin{array}{l}\text { tool } \\
\text { two }\end{array}$ & $\begin{array}{l}\text { toll } \\
\text { tour }\end{array}$ & $\begin{array}{l}\text { rule } \\
\text { cue }\end{array}$ & $\begin{array}{l}\text { roll } \\
\text { cure }\end{array}$ \\
\hline Groupe $n^{\circ} 12$ & $\hat{\Lambda}$ & $\begin{array}{l}\neq 0: \\
x=\end{array}$ & $\begin{array}{l}\text { gull } \\
\text { cub }\end{array}$ & $\begin{array}{l}\text { girl } \\
\text { cab }\end{array}$ & $\begin{array}{l}\text { bud } \\
\text { cup }\end{array}$ & $\begin{array}{l}\text { bird } \\
\text { cap }\end{array}$ \\
\hline Groupe $n^{\circ} 13$ & $\hat{\imath}$ & $\begin{array}{l}\neq 9: \\
=0\end{array}$ & $\begin{array}{l}\text { bun } \\
\text { rub }\end{array}$ & $\begin{array}{l}\text { barn } \\
\text { rob }\end{array}$ & $\begin{array}{l}\text { cup } \\
\text { bus }\end{array}$ & $\begin{array}{l}\text { carp } \\
\text { boss }\end{array}$ \\
\hline Groupe $n^{\circ} 14$ & $\hat{\circ}:$ & $\begin{array}{l}\times 0: \\
\times 01 \\
\end{array}$ & $\begin{array}{l}\text { bud } \\
\text { corn }\end{array}$ & $\begin{array}{l}\text { board } \\
\text { coin }\end{array}$ & cut & caught \\
\hline Groupe $n^{\circ} 15$ & $\begin{array}{l}\text { ai } \\
\text { ei }\end{array}$ & $\begin{array}{l}\neq \text { a aio } \\
\neq \text { eio }\end{array}$ & $\begin{array}{l}\text { tíe } \\
\text { play }\end{array}$ & $\begin{array}{l}\text { tyre } \\
\text { player }\end{array}$ & lie & liar \\
\hline Groupe $n^{\circ} 16$ & $\begin{array}{l}\text { aid } \\
\text { ou }(\bullet u)\end{array}$ & $\begin{array}{l}=\text { eio } \\
\text { ₹ oub }\end{array}$ & $\begin{array}{l}\text { liar } \\
\text { low }\end{array}$ & $\begin{array}{l}\text { Tayer } \\
\text { lower }\end{array}$ & mow & mower \\
\hline Groupe $n^{\circ} \quad 17$ & $\begin{array}{l}\text { au } \\
\text { aid }\end{array}$ & $\begin{array}{l}\neq a u_{0} \\
\neq u_{0}\end{array}$ & $\begin{array}{l}\text { bow } \\
\text { tyre }\end{array}$ & $\begin{array}{l}\text { bower } \\
\text { tower }\end{array}$ & buyer & bower \\
\hline
\end{tabular}

Tableau III. - Les voyelles et les diphtongues anglaises.

[Basic consonantal oppositions in English.]

\begin{tabular}{ll} 
i: & bead \\
i & bid \\
iə & beard \\
ei & bade \\
e & bed \\
Eə & bear \\
ae & bad \\
\hline qi & buy \\
qu & bowed \\
q: & bard \\
o & boss \\
oi & boy \\
o: & board \\
ou & bow \\
\hline u & bull \\
uə & boor \\
u: & booed \\
ə: & bird \\
$\wedge$ & bud \\
ə & cup-board \\
\hline eiə & payer \\
aiə & buyer \\
oiə & employer \\
quə & power \\
ouə & grower
\end{tabular}


concerne les voyelles et diphtongues anglaises. Ces mots sont généralement de durées voisines et ils ne diffèrent, deux à deux, que par des paires minimales.

Les conditions expérimentales sont les suivantes :

- les mots du lexique-référence ont été prononcés par une Anglaise, puis répétés par un Anglais et enregistrés sur cassette;

- l'apprentissage par MicroLEA a été fait à partir de la cassette, par groupe de 8 mots pour se placer dans les conditions dans lesquelles le système travaille effectivement lors d'une leçon (les groupes sont numérotés);

- la reconnaissance se fait également à partir de cette cassette, apprentissage et reconnaissance utilisant le même locuteur.

Les taux de reconnaissance sont établis sur dix essais avec la voix féminine et dix essais avec la voix masculine pour chacun des mots.

Pour les mots du tableau I (groupes no 1, 2, 3, 6, 7, 8), ils sont de $100 \%$ aussi bien pour la voix masculine que pour la voix féminine.

- Pour le groupen 4 : $97 \%$ pour la voix masculine, $92 \%$ pour la voix féminine,

par suite de confusions entre FAN et VAN, LEAVE et LEAF

- Pour le groupe no $5: 92 \%$ pour les 2 voix, par suite de confusions entre SEES et SEETHE, SEETHE et SEES ou BREATHE.

Pour les mots du tableau II (groupes no 1, 2, 3, 4, $5,6,7,9,10,12,13,14,15,16,17)$, on obtient encore $100 \%$ pour les 2 voix. Pour les groupes no 8 et 11 , seulement $95 \%$.

Pour les mots du tableau III, les taux de reconnaissance obtenus sont de $100 \%$ pour tous les groupes.

Il semble que l'on puisse attribuer la plupart de ces confusions à une mauvaise acquisition (soit en apprentissage, soit en reconnaissance) lorsque le mot commence (ou finit) par un phonème peu "énergétique » tel que /f/ ou /1/. En effet, le processus d'acquisition est déclenché (ou arrêté) lorsque l'énergie recueillie par les filtres est supérieure (ou inférieure) à un seuil déterminé expérimentalement. On minimise effectivement ces confusions en prenant en compte systématiquement quelques échantillons supplémentaires en début et en fin de mot.

Par contre, pour les confusions portant sur des phonèmes autres que le premier ou le dernier du mot, aucun remède n'a été trouvé. Les tests effectués avec une analyse à partir d'un plus grand nombre de filtres (16 au lieu de 8) n'ont pas apporté l'amélioration escomptée.

Pour chaque mot à reconnaître, le processus de reconnaissance inscrit sur l'écran de visualisation les trois mots du lexique déterminés comme les plus proches du mot prononcé au sens du score $S\left({ }^{1}\right)$ de la comparaison dynamique. Ces trois mots sont classés

$\left.{ }^{1}\right)$ (Voir définition mathématique en annexe). dans l'ordre des taux de dissemblance croissants et les valeurs numériques des scores sont fournies. La connaissance de ces scores est intéressante car elle permet de montrer la cohérence des résultats obtenus. On constate que le taux de dissemblance est petit lorsque le mot du lexique classé en tête est identique au mot à reconnaître. Les scores deviennent relativement peu élevés lorsqu'il s'agit de deux mots phonétiquement voisins (VAN, FAN ou LEAVE, LEAF, etc...). Par contre, les taux de dissemblance obtenus deviennent très grands lorsque les mots comparés sont très différents. Ceci apparaît sur les exemples typiques des tableaux matriciels IV, V, VI, VII où les éléments diagonaux sont effectivement les plus petits et où figurent peu d'éléments non nuls loin de la diagonale.

Les mots phonétiquement voisins (tels que VAN et FAN du tableau IV) étant écrits l'un derrière l'autre dans les lexiques, il en résulte effectivement que les scores classés deuxième dans ces tableaux font surtout apparaître des éléments proches de la diagonale. Les scores classés troisième apparaissent un peu plus loin.

Ainsi qu'il a déjà été dit, la phonématique de l'anglais n'est pas le seul problème rencontré par les anglicistes. Tout aussi difficile est l'acquisition d'un bon accent tonique. Or, ceci est fondamental. En effet, ainsi que l'indique Martinet A. [26] : «Un mot mal accentué n'est pas compris même si les phonèmes qui le composent sont prononcés à la perfection ».

L'expérience montre que si on "apprend " à MicroLEA des prononciations erronées du point de vue de l'accent tonique, il lui est ensuite possible de reconnaître ces fautes. Ceci a été vérifié sur des mots (Tableau VIII) pour lesquels le " pattern » accentuel est un signe indiquant la catégorie grammaticale à laquelle appartient le mot (dans les exemples choisis soit nom, soit verbe). Les scores indiqués au tableau matriciel IX montrent clairement qu'une faute d'accent tonique entraîne des taux de dissemblance, mesurés par MicroLEA, très élevés.

L'ensemble de ces résultats montrent les capacités de MicroLEA à différencier des mots phonétiquement très voisins, et par là-même à détecter les fautes couramment faites par l'apprenti angliciste s'exerçant par exemple à prononcer les consonnes interdentales $|\theta|$ et $|\grave{j}|$, ou bien les occlusives, ou encore toutes les diphtongues qui n'existent pas en français, ainsi que les innombrables fautes d'accent tonique.

Pour conclure sur l'utilité de MicroLEA, il importe de tester ses capacités à détecter ces mêmes fautes lorsque le locuteur effectuant la reconnaissance est différent de celui qui a réalisé l'apprentissage. Ce point est essentiel car ce sont là précisément les conditions normales de fonctionnement du système si on veut l'utiliser en didactique des langues.

Les tests ont été réalisés sur un locuteur francophone possédant déjà la maîtrise de l'anglais écrit et s'exprimant oralement de façon très correcte sur le plan syntaxique, mais non parfaite quant à l'accent. 
Tableau IV. - Exemple typique de tableau matriciel donnant les scores de reconnaissance pour des exemples d'opposition consonantique.

[Typical matricial presentation of recognition scores for examples of consonantal oppositions.]

\begin{tabular}{|c|c|c|c|c|c|c|c|c|}
\hline mot & VAN & FAN & LEAVE & LEAF & RISE & RICE & SEIZE & CEASE \\
\hline VAN & 13 & 26 & & 78 & & & & \\
\hline FAN & 38 & 22 & & & & & 81 & \\
\hline LEAVE & & & 13 & 50 & 65 & & & \\
\hline LEAF & & & 49 & 12 & & & 61 & \\
\hline RISE & & & 68 & & 7 & 47 & & \\
\hline RICE & & & & 63 & 49 & 9 & & \\
\hline SEIZE & & & & 59 & & & 6 & 32 \\
\hline CEASE & & & & 68 & & & 28 & 8 \\
\hline
\end{tabular}

Tableau V. - Exemple typique de tableau matriciel donnant les scores de reconnaissance pour des exemples d'opposition consonantique.

[Typical matricial presentation of recognition scores for examples of consonantal oppositions.]

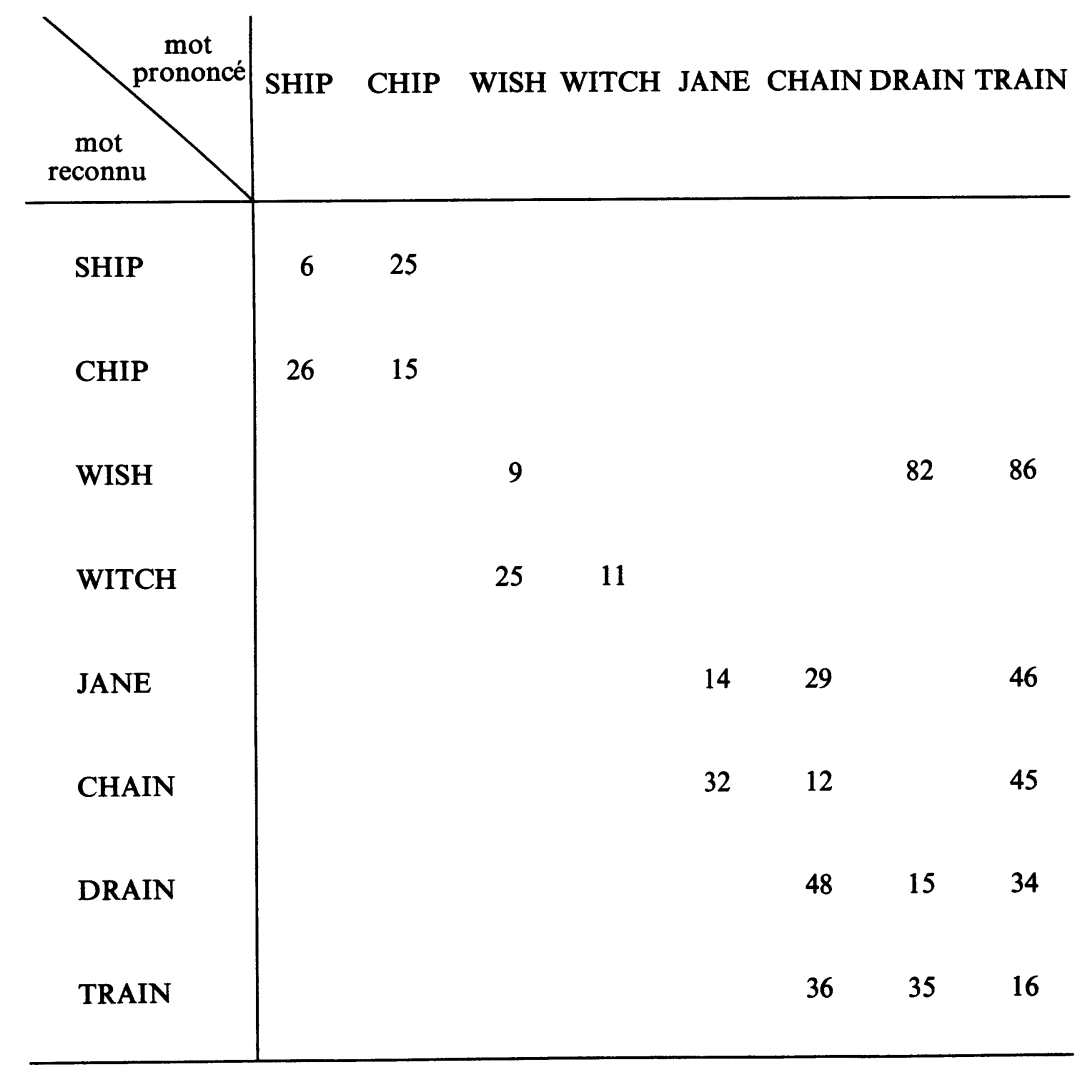


Tableau VI. - Exemple typique de tableau matriciel donnant les scores de reconnaissance pour des exemples d'opposition vocalique.

[Typical matricial presentation of recognition scores for examples of vocalic oppositions.]

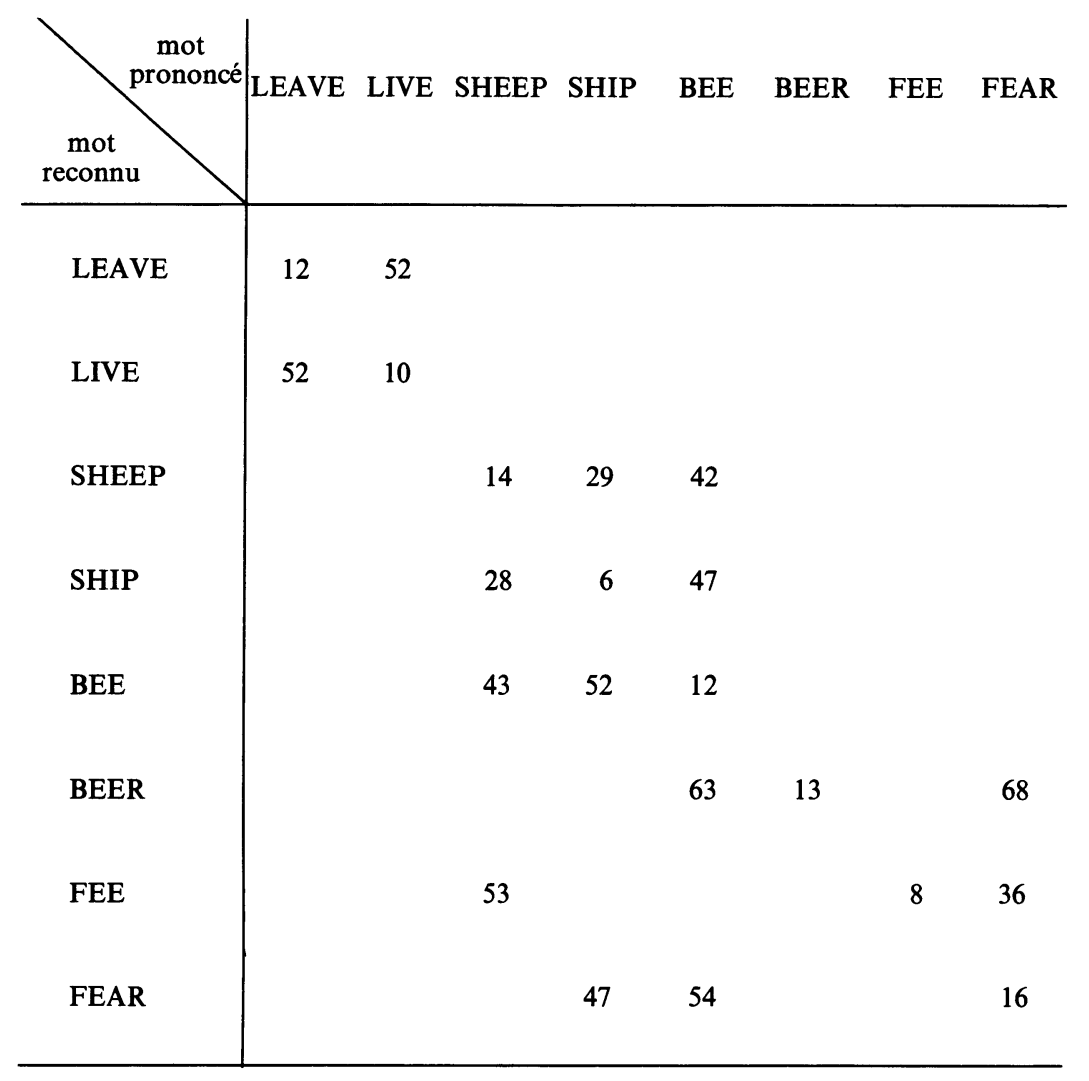

Tableau VII. - Exemple typique de tableau matriciel donnant les scores de reconnaissance pour des exemples d'opposition vocalique.

[Typical matricial presentation of recognition scores for examples of vocalic oppositions.]

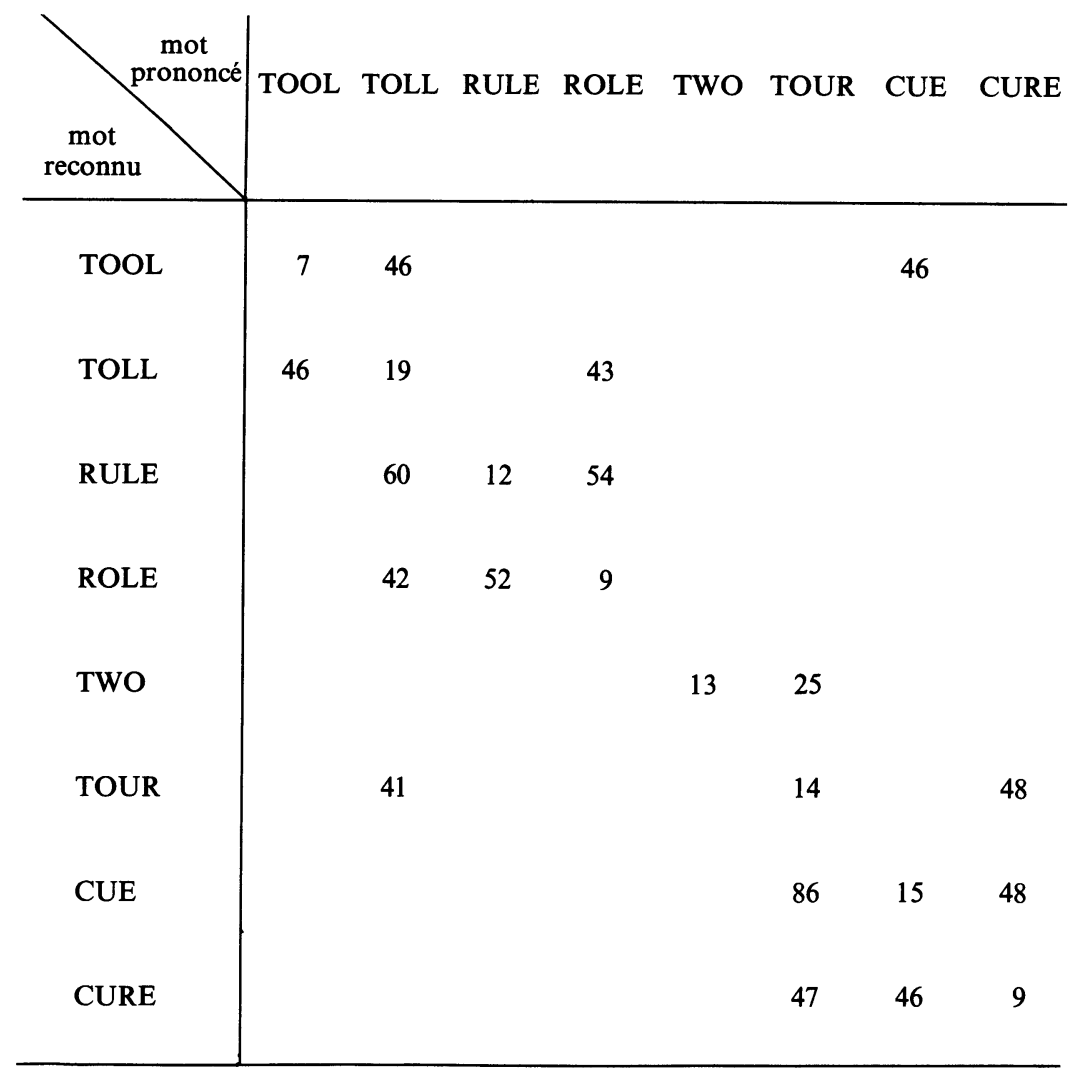


Tableau VIII. - Liste de mots pour lesquels la place de l'accent tonique est un indice de la catégorie grammaticale.

[Typical words in which the stressed syllable is a grammatical information.]

(sur la première syllabe $\rightarrow$ nom)

(sur la dernière syllabe $\rightarrow$ verbe)

Absent
Contract
Contrast
Export
Object
Perfect
Permit
Produce
Progress
Protest
Rebel
Record
Refuse
Subject
Survey

L'expérience montre qu'après plusieurs répétitions, sa prononciation se rapproche suffisamment de celle du "modèle" pour que le système "reconnaisse" le mot testé. Il est intéressant de noter les scores délivrés par le système dans les deux cas suivants :

- lorsque apprentissage et reconnaissance sont exécutés à partir de la " cassette-leçon ", donc avec la même voix;

- lorsque l'apprentissage est réalisé à partir de la " cassette-leçon » (enregistrement du locuteur anglophone) et la reconnaissance effectuée par le locuteur francophone essayant d'imiter de son mieux la prononciation " modèle ".
Les résultats correspondants figurent dans le tableau X.

La partie supérieure de ce tableau correspond au premier cas et les commentaires qui peuvent être faits sont analogues à ceux correspondant aux tableaux IV, V, VI, VII. La partie inférieure donne les taux de dissemblance obtenus dans le deuxième cas, après plusieurs essais infructueux et en nombre variable suivant les difficultés de prononciation à surmonter. Ces scores finaux demeurent toujours plus élevés que ceux obtenus dans le premier cas, mais restent néanmoins groupés le long de la diagonale du tableau matriciel.

\section{Tests d'efficacité sur le déroulement d'une leçon.}

Là encore, les enseignants-chercheurs du C.R.A.P.E.L. nous ont apporté leur aide en enregistrant une cassette qui nous a permis de réaliser plusieurs exemples de leçon. Les mots choisis figurent dans le tableau XI.

Chacun de ces mots a été prononcé par trois anglophones : un Britannique, une Australienne et une Américaine. Les versions erronées ont été fournies par quatre étudiants francophones (deux garçons et deux filles) (Tableau XII).

L'apprentissage par MicroLEA a été réalisé à partir des voix britannique et américaine, pour les " modèles", et par les voix des étudiants pour les versions entachées d'erreurs.

Lors du déroulement de la leçon, ont joué le rôle d'élèves :

- l'enregistrement de l'Australienne,

- une Française professeur d'anglais,

- un Canadien anglophone,

- l'enregistrement des étudiants,

- deux " cobayes" francophones (un homme et une femme).

Tableau IX. - Exemple typique de tableau matriciel donnant les scores de reconnaissance pour des exemples de mots pour lesquels la place de l'accent tonique est un indice de la catégorie grammaticale.

[Typical recognition scores for examples of words whose stressed syllable is a grammatical information.]

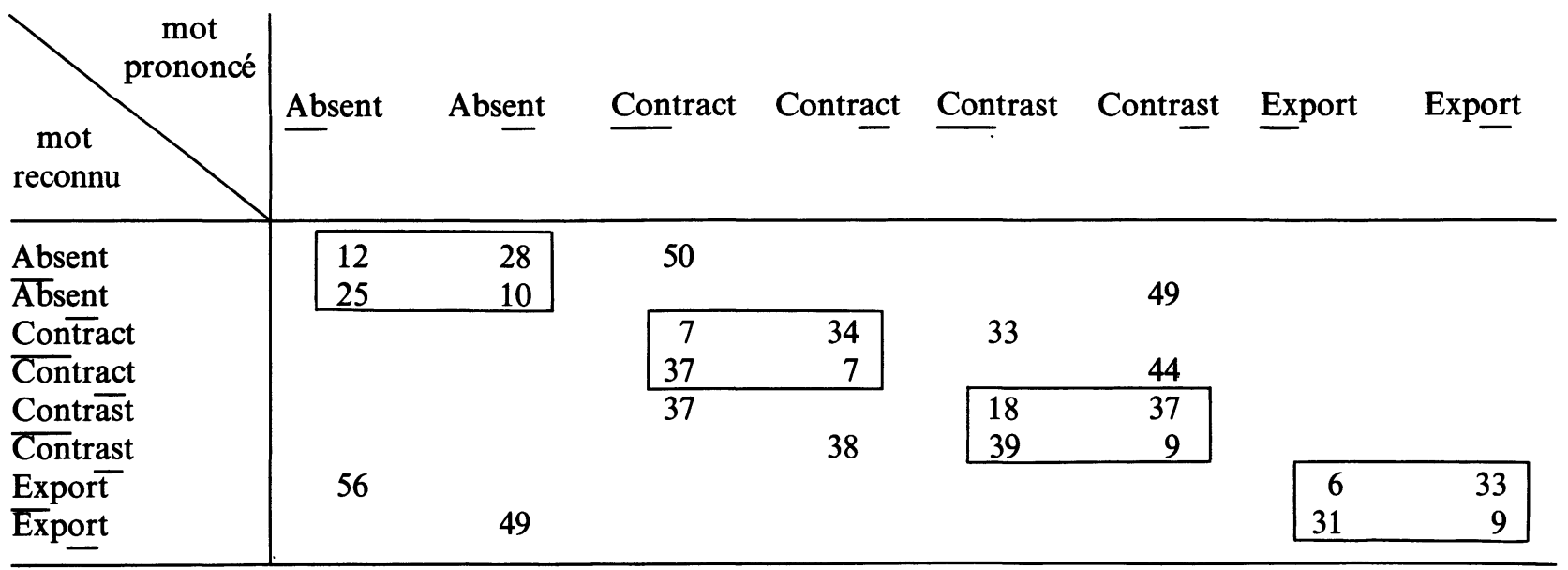


Tableau X. - Comparaison des scores de reconnaissance entre " professeur et élève " (voir texte).

[Typical recognition scores for « teacher » and « student » as compared to each other (see the text).]

\begin{tabular}{|c|c|c|c|c|c|c|c|c|}
\hline \multirow{2}{*}{ mot mot } & \multicolumn{2}{|c|}{$\underline{\text { Professeur/Professeu }}$} & \multirow[b]{2}{*}{ Force } & \multirow[b]{2}{*}{ Fourth } & \multirow[b]{2}{*}{$\operatorname{Sin}$} & \multirow[b]{2}{*}{ Thin } & \multirow[b]{2}{*}{ Breeze } & \multirow[b]{2}{*}{ Breathe } \\
\hline & Sees & Seethe & & & & & & \\
\hline Sees & 10 & 32 & 67 & & & & & \\
\hline Seethe & 25 & 14 & & & & & & 49 \\
\hline Force & & & 14 & 35 & & & 63 & \\
\hline Fourth & & & 35 & 12 & & 80 & & \\
\hline Sin & & 47 & & & 15 & 49 & & \\
\hline Thin & & & & & 47 & 18 & & \\
\hline Breeze & 50 & & & & & & 8 & 40 \\
\hline Breathe & & 56 & & & & & 37 & 6 \\
\hline
\end{tabular}

\begin{tabular}{|c|c|c|c|c|c|c|c|c|}
\hline \multirow{2}{*}{ reconnu } & \multicolumn{2}{|c|}{ Professeur/Elève } & \multirow[b]{2}{*}{ Force } & \multirow[b]{2}{*}{ Fourth } & \multirow[b]{2}{*}{ Sin } & \multirow[b]{2}{*}{ Thin } & \multirow[b]{2}{*}{ Breeze } & \multirow[b]{2}{*}{ Breathe } \\
\hline & Sees & Seethe & & & & & & \\
\hline Sees & 32 & 34 & & & 55 & & & \\
\hline Seethe & 43 & 36 & & & 62 & & & \\
\hline Force & & & 46 & 49 & & 100 & & \\
\hline Fourth & & & 43 & 41 & & & 80 & \\
\hline $\operatorname{Sin}$ & & & & & 50 & 52 & & \\
\hline Thin & & & & & 70 & 56 & & 76 \\
\hline Breeze & 57 & & & & & & 51 & 57 \\
\hline Breathe & & 77 & & & & & 64 & 60 \\
\hline
\end{tabular}

Les résultats sont les suivants :

MicroLEA a jugé bonne la prononciation de l'Australienne, du Canadien et du professeur d'anglais, a reconnu correctement les fautes des étudiants (sans aucune erreur de la part du système) a permis aux « cobayes " d'améliorer leur prononciation en répétant les mots jusqu'à ce que le système délivre le message BON plusieurs fois de suite.

Tableau XI. - Lexique correspondant à une leçon-test.

[Lexical example corresponding to a tested lesson.]

Solid
Enjoyable
Tripod
Agregate
Warehouse
Outnumber
Theories
Physical
Laser
Prepare
Comfortable
Assistant
Measure

Pour évaluer de façon plus "quantitative" l'efficacité du système, voici un exemple de leçon et, pour chacun des mots étudiés (TRIPOD, LASER, COMFORTABLE), un tableau permettant de comparer les scores obtenus par les "professeurs" et par les " élèves". Les conditions expérimentales sont les suivantes :

- Pour TRIPOD, l'apprentissage par MicroLEA a été réalisé à partir des voix britannique et américaine pour les modèles et par les voix d'un étudiant et d'une étudiante pour les versions entachées d'erreurs. Ces erreurs sont soit seulement une mauvaise prononciation du $\underline{i}(|\mathrm{i}|$ au lieu de $\mid$ ai $\mid)$, soit en plus une faute d'accent tonique.

La reconnaissance a été effectuée à partir de l'enregistrement des deux anglophones, de celui des étudiants, ainsi que par ces mêmes étudiants essayant de corriger leurs fautes et de se rapprocher le plus possible des " modèles ".

Les résultats correspondants sont rassemblés dans le tableau XIII. On constate de nouveau que les scores des professeurs sont nettement meilleurs que ceux obtenus par les élèves lorsqu'ils parviennent à se rapprocher suffisamment de la prononciation correcte fournie par les modèles. 
Tableau XII. - Exemple typique des fautes de prononciation commises par des élèves francophones sur un lexique de mots anglais.

[Typical mispronunciations by french native speakers in some English words.]

\begin{tabular}{|c|c|c|c|}
\hline Premier élève & Deuxième élève & Troisième élève & Quatrième élève \\
\hline so'lid & so'lid & so'lid & solid \\
\hline enjōyable & enjōyable & enjoyable & enjoyyable \\
\hline tripod & tri’pod & tripod & tri'pod \\
\hline 'agregate & $a^{\prime}$ gregate & a'gregate & agre'gate \\
\hline ware'house & wărehouse & warehouse & warehouse \\
\hline outnumber & outnūmber & outnumber & outnūmber \\
\hline theories & theories & the'ories & theories \\
\hline physi'cal & phy'sical & physical & physical \\
\hline laser & laser & lassẹer & laser \\
\hline 'prepare & 'prepare & 'prepare & prepare \\
\hline comfortable & com'fortable & comfortable & comfor'table \\
\hline ássistant & assis'tant & assistant & 'assistant \\
\hline meásure & mea'sure & measure & measure \\
\hline
\end{tabular}

Les apostrophes indiquent les fautes d'accent tonique et les tirets les fautes de prononciation sur les phénomènes.

En cas de bonne prononciation phonétique mais d'absence d'accent tonique, il n'y a ni apostrophe, ni tiret.

Tableau XIII. - Exemple typique de tableau matriciel donnant les scores de reconnaissance obtenus par deux professeurs et deux élèves prononçant le mot anglais TRIPOD.

[Typical matricial presentation of recognition scores obtained by two teachers and two learners in pronuncing the English word TRIPOD.]

\begin{tabular}{|c|c|c|c|c|c|c|}
\hline \multirow{3}{*}{ Reconnaissance } & \multirow{3}{*}{$\begin{array}{c}\text { Anglais } \\
6\end{array}$} & \multirow{3}{*}{$\begin{array}{c}\text { Américaine } \\
32\end{array}$} & Elève $(F)$ & Elève $(\mathrm{H})$ & Elève $(F)$ & Elève $(H)$ \\
\hline & & & \multicolumn{2}{|c|}{ Faute sur le $\underline{\mathrm{i}}$} & \multicolumn{2}{|c|}{$\begin{array}{l}\text { Faute sur le } \mathrm{i} \\
+ \text { accent tonique }\end{array}$} \\
\hline & & & & 41 & & \\
\hline Américaine & 44 & 7 & 58 & & & \\
\hline $\begin{array}{l}\text { Elève }(\mathrm{F}) \\
\text { Bonne prononciation }\end{array}$ & 49 & 45 & 81 & & & \\
\hline $\begin{array}{l}\text { Elève }(\mathrm{H}) \\
\text { Bonne prononciation }\end{array}$ & 34 & 28 & & 52 & & \\
\hline $\begin{array}{l}\text { Elève }(F) \\
\text { Faute sur le } \mathrm{i}\end{array}$ & 47 & & 40 & 41 & & \\
\hline $\begin{array}{l}\text { Elève }(\mathrm{H}) \\
\text { Faute sur le } \mathrm{i}\end{array}$ & 49 & & & 21 & 72 & \\
\hline $\begin{array}{l}\text { Elève }(F) \\
\text { Faute sur le } \mathrm{i} \\
+ \text { accent toñique }\end{array}$ & & & 40 & & 22 & 30 \\
\hline $\begin{array}{l}\text { Elève }(\mathrm{H}) \\
\text { Faute sur le } \mathrm{i} \\
+ \text { accent toñique }\end{array}$ & & & 40 & & 39 & 16 \\
\hline
\end{tabular}


Avant de parvenir à ce résultat, lorsque les élèves commettent les erreurs connues du système, les scores qu'ils obtiennent montrent d'une part que la faute a été identifiée et d'autre part que le système est plus sensible à la nature de l'erreur qu'au type de la voix (masculine ou féminine).

- Pour LASER, les conditions expérimentales sont les mêmes, à ceci près qu'on a utilisé pour l'apprentissage un troisième "modèle » fourni par la voix de l'Australienne. Les fautes sont soit une mauvaise prononciation du a $(|\mathrm{e}|$ au lieu de $\mid$ ei $\mid)$, soit une faute d'accent tonique.

Les résultats, rapportés dans le tableau XIV, sont analogues aux précédents.

- Pour COMFORTABLE, les fautes sont soit une mauvaise prononciation du a (| ei | au lieu de $\mid$ ae $\mid)$ avec, en plus, l'accent toniqūe placé sur la dernière syllabe au lieu de la première, soit seulement une faute d'accent tonique (la deuxième syllabe accentuée au lieu de la première).

Il faut noter, à propos des résultats du tableau $\mathrm{XV}$, que les élèves ont eu beaucoup de mal à bien prononcer ce mot et que les scores indiqués ont été obtenus après de nombreux essais.

\section{Conclusion.}

Pour conclure sur l'intérêt pédagogique de cet appareil, il faudrait attendre le verdict des utilisateurs, c'est-à-dire des enseignants d'anglais. Cependant, les tests effectués au laboratoire montrent d'ores et déjà que le système fonctionne correctement dans la plupart des cas.

Cela tient à l'algorithme de reconnaissance fondé sur la comparaison dynamique, sur l'utilisation en apprentissage de plusieurs voix de référence. De plus, il faut noter qu'en réalité la comparaison se fait chaque

Tableau XIV. - Exemple typique de tableau matriciel donnant les scores de reconnaissance obtenus par deux professeurs et deux élèves prononçant le mot anglais LASER.

[Typical matricial presentation of recognition scores obtained by two teachers and two learners in pronuncing the English word LASER.]

\begin{tabular}{|c|c|c|c|c|c|c|c|}
\hline \multirow{3}{*}{$\begin{array}{l}\text { Reconnaissance } \\
\text { Anglais }\end{array}$} & \multirow{3}{*}{$\begin{array}{c}\text { Anglais } \\
11\end{array}$} & \multirow{3}{*}{\begin{tabular}{|c} 
Américaine \\
41
\end{tabular}} & \multirow{3}{*}{ Australienne } & Elève $(F)$ & Elève $(\mathrm{H})$ & Elève $(F)$ & Elève $(\mathrm{H})$ \\
\hline & & & & \multicolumn{2}{|c|}{ Faute sur le a } & \multicolumn{2}{|c|}{$\begin{array}{l}\text { Faute d'accent } \\
\text { tonique }\end{array}$} \\
\hline & & & & & & 50 & \\
\hline Américaine & 38 & 11 & 40 & & & & \\
\hline Australienne & 45 & 33 & 6 & & & & \\
\hline $\begin{array}{l}\text { Elève }(F) \\
\text { Bonne } \\
\text { prononciation }\end{array}$ & 31 & 39 & & & & 47 & \\
\hline $\begin{array}{l}\text { Elève }(\mathrm{H}) \\
\text { Bonne } \\
\text { prononciation }\end{array}$ & 28 & 48 & & & & 59 & \\
\hline $\begin{array}{l}\text { Elève }(F) \\
\text { Faute sur le a }\end{array}$ & 54 & & & 12 & 44 & & \\
\hline $\begin{array}{l}\text { Elève }(\mathrm{H}) \\
\text { Faute sur le a }\end{array}$ & & & & 41 & 28 & 71 & \\
\hline $\begin{array}{l}\text { Elève }(\mathrm{F}) \\
\text { Faute d'accent } \\
\text { tonique }\end{array}$ & & & & 57 & & 17 & 54 \\
\hline $\begin{array}{l}\text { Elève }(\mathrm{H}) \\
\text { Faute d'accent } \\
\text { tonique }\end{array}$ & & & & & 71 & 37 & 22 \\
\hline
\end{tabular}


Tableau XV. - Exemple typique de tableau matriciel donnant les scores de reconnaissance obtenus par deux professeurs et deux élèves prononçant le mot anglais COMFORT ABLE.

[Typical matricial presentation of recognition scores obtained by two teachers and two learners in pronuncing the English word COMFORTABLE.]

\begin{tabular}{|c|c|c|c|c|c|c|c|}
\hline Apprentissage & & & & Elève $(F)$ & Elève $(\mathrm{H})$ & Elève $(F)$ & Elève $(\mathbf{H})$ \\
\hline Reconnaissance & Anglais & Américaine & Australienne & \multicolumn{2}{|c|}{$\begin{array}{l}\text { Faute d'accent } \\
\text { tonique }\end{array}$} & \multicolumn{2}{|c|}{$\begin{array}{l}\text { Faute d'accent } \\
\text { tonique } \\
+ \text { faute sur le a }\end{array}$} \\
\hline Anglais & 10 & 40 & & & 43 & & \\
\hline Américaine & & 14 & 50 & & & & \\
\hline Australienne & & 48 & 10 & & & & 67 \\
\hline $\begin{array}{l}\text { Elève }(F) \\
\text { Bonne } \\
\text { prononciation }\end{array}$ & & 37 & & 39 & & & 46 \\
\hline $\begin{array}{l}\text { Elève }(\mathrm{H}) \\
\text { Bonne } \\
\text { prononciation }\end{array}$ & & 39 & 54 & & & & 51 \\
\hline $\begin{array}{l}\text { Elève }(F) \\
\text { Faute d'accent } \\
\text { tonique }\end{array}$ & & & & 23 & 29 & 41 & \\
\hline $\begin{array}{l}\text { Elève }(\mathbf{H}) \\
\text { Faute d'accent } \\
\text { tonique }\end{array}$ & & & & 36 & 10 & & 33 \\
\hline $\begin{array}{l}\text { Elève }(F) \\
\text { Faute sur le a } \\
+ \text { accent tonique }\end{array}$ & & & & & 40 & 23 & 31 \\
\hline $\begin{array}{l}\text { Elève }(\mathrm{H}) \\
\text { Faute sur le a } \\
+ \text { accent tonique }\end{array}$ & & & & 43 & & 39 & 22 \\
\hline
\end{tabular}

fois entre le mot prononcé et un très petit nombre de mots (ceci quel que soit le volume total de la leçon).

Un tel système ne peut pas reconnaître toutes les erreurs possibles, mais lorsque l'élève fait une faute inconnue du processus, celui-ci se borne à délivrer le message «Mauvais » et la réaudition de la voix du professeur permet à l'élève de s'auto-corriger.

Enfin, il convient de remarquer que la conception de la leçon peut évoluer en fonction de l'âge des élèves et de l'objectif envisagé. On peut imaginer un prolongement aux premiers exercices décrits dans ce travail : lorsque l'étape d'acquisition de la prononciation est franchie, le dialogue entre la machine et l'élève peut être modifié de la façon suivante : chaque mot est d'abord enregistré en français et l'élève doit le dire dans la langue étudiée. L'élève n'entend la traduction enregistrée par le professeur que s'il a fait une erreur, soit de traduction, soit de prononciation. Ainsi seraient à la fois testées la mémorisation et l'acquisition de la prononciation correcte du vocabulaire étudié.

D'autres développements sont possibles. Par exemple : enregistrer la voix de l'élève et inclure dans le programme son écoute automatique, ce qui lui permettrait de mieux prendre conscience de son erreur de prononciation.

Une autre point mérite d'être souligné : c'est la possibilité pour l'étudiant de travailler seul. Un tel système ne se substitue certes pas complètement au professeur (de toutes manières, l'organisation des leçons incombe à l'enseignant) et il ne permet pas de détecter toutes les fautes de prononciation (en particulier il serait sans doute bon de prendre en compte 
des informations prosodiques telle que la hauteur de la voix). Toutefois, en son état actuel, MicroLEA constitue une ébauche intéressante en tant qu'outil de travail pour un apprentissage autodidactique, surtout à l'usage d'adultes non débutants dans la langue et cherchant à améliorer leur prononciation.

\section{Remerciements.}

Les auteurs tiennent à remercier les chercheurs du Centre de Recherches et d'Applications Pédagogiques en Langues de Nancy, qui les ont aidés à mener à bien ce travail.

\section{Bibliographie}

[1] SHERwood, B. A., Int. J. Man-Machine Studies 10 (1978) 669.

[2] Simon, J. C., L'éducation et l'informatisation de la société. Rapport au Président de la République Ed. par Fayard (1981).

[3] Bestougeff, H., Thèse d'Etat Université de Paris VII (1970).

[4] Nelles, R. et SenneKamp, M., Gr. K. G. 18/4 (1977) 37.

[5] Giorgetti, M. T. et Lamotte, M., Actes des XIe Journées d'Etudes sur la Parole (1980) 202.

[6] NordmanN, B. J., A comparative study of some visual speech displays Rapport de contrat (1971) Univ. Illinois. USA.

[7] Kalikow, D. W. et Swets, I. A., Proc. of the I.E.E.E. Fall Electronics Conference (1971) 19.

[8] Harada, K. I., Annual Bulletin Research Institute of Logopedics and Phoniatrics 5 (1971) 51.

[9] Lebras, J., Thèse de 3e Cycle Université de Rennes II (1981).

[10] Bellissant, C., Thèse d'Etat Université de Grenoble (1978).

[11] Mas, M. T., Vigneron, M. J., Lamotte, M., Gr. $K$. G. 18/4 (1977) 97.

[12] Vigneron, C., Thèse de Doctorat d'Université Université de Nancy I (1978).

[13] Janot-Giorgetti, M. T. et Lamotte, M., Gr. K. G. 23/2 (1982) 81

Annexe. - Méthode de reconnaissance par «comparaison dynamique $":$ principes généraux.

\section{Introduction.}

Nous ne nous étendrons pas sur la méthode en ellemême car elle a fait l'objet de nombreux ouvrages et publications (cf. la bibliographie) mais, après avoir posé le problème et énoncé le principe de sa résolution, nous montrerons la simplification qui en est faite ici dans son application à la reconnaissance de la parole.

Soit un système dont l'état est repéré par une variable scalaire (ou vectorielle) qui évolue sous l'effet de décisions, et soit à trouver dans l'espace des états possibles du système envisagé, une trajectoire optimale pour une certaine fonction de valeur, en choisissant convenablement les variables de contrôle.

Ce type de problème se rencontre aussi bien en physique (exemple : recherche d'une trajectoire optimale pour un mobile devant rester en contact avec une surface) qu'en économie [1] et peut être résolu par une technique mathématique développée initialement par
[14] Duroc, M., Thèse de 3e Cycle Université de Nancy I (1978).

[15] Bremont, J., Thèse d'Etat Université de Nancy I (1975).

[16] Gold, B., Blankenship, P. E., McAulay, R. J., On Acoustics, Speech and Signal Processing. I.E.E.E. Trans. 29/1 (1981) 13.

[17] Lamotte, M. et Mas, M. T., Méc. Mat. Elec. 336 (1976) 3.

[18] Janot-Giogetri, M. T., Thèse d'Etat Université de Nancy I (1982).

[19] Bars, J. F., Gresser, J. Y., Querre, M., Actes des IVe Journées d'Etudes sur la Parole (1973) 327.

[20] Haton, J. P., Thèse d'Etat Université de Nancy I (1974).

[21] Sakoe, H. et Chiba, S., Proc. of the 7th International Congress on Acoustics, Budapest (1971).

[22] Vintsiuk, T. K., Kibernetika 1 (1968) 81.

[23] CARTON, F., Introduction à la phonétique du français (Ed. par Bordas) 1979.

[24] Malmberg, B., La phonétique (Ęd. par P.U.F.) 1975.

[25] Adamczewski, H. et Keen, D., Phonétique et Phonologie de l'anglais contemporain (Ed. par Armand Colin) 1979.

[26] Martinet, A., Eléments de linguistique générale (Ed. par Armand Colin) 1974.

l'équipe de Bellman R. : la programmation dynamique. Cette méthode est fondée sur le «principe d'optimalité », énoncé comme suit dans l'ouvrage de Bellman R. [2] :

"Une politique optimale est telle que, quels que soient l'état initial et la décision initiale, les décisions suivantes doivent constituer une politique optimale par rapport à l'état résultant de la première décision. ॥

En reconnaissance de la parole, il s'agit d'identifier un mot inconnu c'est-à-dire déterminer quel est le mot d'un lexique de référence auquel il ressemble le plus. Cela revient à minimaliser un «taux de dissemblance » entre deux formes.

\section{Application à la reconnaissance de la parole.}

Ainsi qu'il a été indiqué, le signal vocal émis par le locuteur est analysé par un banc de $n$ filtres qui évalue, toutes les $20 \mathrm{~ms}$, sous forme numérique, l'état du spectre du signal vocal. Ces données sont transmises au calculateur sous la forme "d'échantillons" à n composantes, si bien qu'un mot $i$ de durée $T_{i}$ échan- 
tillonné $N_{i}$ fois $\left(N_{i}=50 T_{i}\right)$ sera représenté par la matrice $R_{i}$.

$$
R_{i}=\left[x_{1}, x_{2}, \ldots, x_{j}, \ldots, x_{n}\right]
$$

avec $x_{j}$ vecteur-colonne d'éléments $x_{j}^{k}(k$ variant de 1 à $n$ ) où $x_{j}^{k}$ est la réponse de $k$-ième filtre à l'instant $t=j \times 20 \mathrm{~ms}$.

Durant la phase dite "d'apprentissage", il est possible d'emmagasiner, en mémoire du calculateur, un nombre $L$ de formes sonores qui constitueront le lexique de référence. Chacune des formes est caractérisée par la matrice $R$ de dimensions $n \times N, n$ étant égal au nombre de filtres constituant l'analyseur et $N$ étant variable.

Lors de la reconnaissance, l'acquisition se fera de la même façon, c'est-à-dire que le mot prononcé sera représenté par la matrice :

$$
M=\left[Y_{1}, Y_{2}, \ldots, Y_{i} \ldots Y_{p}\right]
$$

$p$ étant le nombre d'échantillons constituant le mot prononcé et $Y_{i}$ étant un vecteur-colonne d'éléments $y_{i}^{k}$ ( $k$ variant de 1 à $n$ ).

Il s'agit ensuite de rechercher la plus grande ressemblance (ou encore la dissemblance minimale) entre le mot prononcé et un des mots du lexique. Pour ce faire, chaque échantillon $Y_{i}$ du mot à reconnaître est comparé aux échantillons $X_{j}$ du mot du lexique, en partant du début des mots et en progressant vers leur fin.

On peut représenter schématiquement cette démarche sur un diagramme bidimensionnel (Fig. 5) où l'on place en abscisse des points figuratifs des échantillons $X_{j}$ pour un mot du lexique donné, et en ordonnée des points figuratifs analogues $Y_{i}$ pour le mot prononcé.

L'ensemble forme un graphe dont chaque nœud $(j, i)$ tel que $C\left(X_{j}, Y_{i}\right)$ est l'image de la comparaison entre $X_{j}$ et $Y_{i}$ définie ainsi :

$$
\mathrm{d}\left(X_{j}, Y_{i}\right)=\mathrm{d}(i, j)=\sum_{k=1}^{n}\left|Y_{i}^{k}-X_{j}^{k}\right| .
$$

La recherche du taux de dissemblance minimal revient à chercher le chemin optimal en "coût" entre $A$ et $B$ dans le graphe composé d'arcs qui sont orientés dans le sens de la séquence d'échantillons constituant les mots. Notons qu'en chaque point du maillage peuvent arriver plusieurs arcs.

La détermination du chemin optimal par programmation dynamique consiste à se définir une fonction coût, calculée au point d'arrivée de l'arc, à partir de la fonction coût du point d'origine $\mathrm{d}\left(X_{1}, Y_{1}\right)$. Puis, il faut choisir, en chaque point, l'arc par lequel il convient d'arriver pour rendre la fonction coût optimale (c'est-à-dire, dans ce cas, le taux de dissemblance minimale). Ce choix constitue la "fonction retour".

La procédure se déroule étape par étape (de A vers B) et les résultats intermédiaires (fonction coût et choix de l'arc) sont conservés. Il suffit ensuite de parcourir le

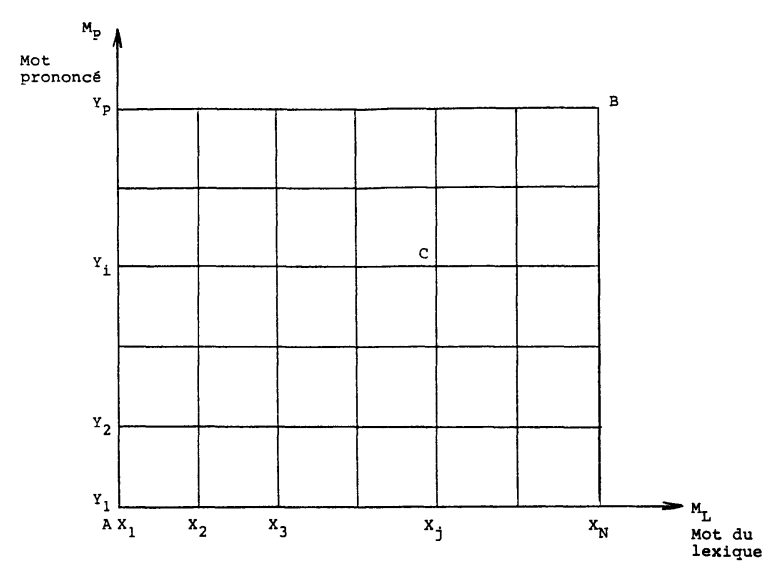

Fig. 5. - Représentation schématique bidimensionnelle du processus de reconnaissance par programmation dynamique.

[A two dimensional picture of speech recognition procedure using dynamic programming.]

graphe en sens inverse, de B vers A, en utilisant la fonction retour pour découvrir le chemin optimal.

En reconnaissance de mots isolés, cette procédure est assez longue. En outre, cela conduit à comparer certains couples d'échantillons qui, à priori, n'ont pas lieu de l'être (exemple : $X_{1}$ et $Y_{p}$ ). On préfère donc se définir à chaque étape, c'est-à-dire pour chaque échantillon du mot à reconnaître, un nombre de chemins élémentaires raisonnables à l'intérieur d'une cellule donnée (voir Fig. 6) où l'on prend seulement en compte des échantillons chronologiquement voisins dans un mot de référence et le mot à reconnaître. Cette méthode peut être désignée sous le nom de « comparaison dynamique $"$.

On choisit celui qui donne le coût optimal, c'est-àdire celui pour lequel la distance $\mathrm{d}(i, j)$ est minimale. Soit, à l'étape $k$ :

$$
\begin{array}{r}
s_{k}=\operatorname{Min}[\mathrm{d}(i, j+1), \mathrm{d}(i+1, j+1), \mathrm{d}(i+1, j+2), \\
\mathrm{d}(i+2, j+1), \mathrm{d}(i+1, j)]
\end{array}
$$

avec $s_{1}=\mathrm{d}(1,1)$.

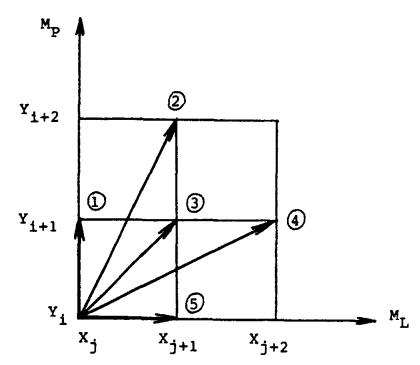

Chemins 1: répétition dans $M_{p}$ 2 : omission dans $M_{L}$ 3 : avance normale 4: omission dans $M_{P}$ 5 : répétition dans $M_{L}$

Fig. 6. - Illustration du procédé de limitation des chemins de comparaison pris en compte dans le processus de reconnaissance.

[Pictural description of the cut-off procedure used to restrict the number of reasonable comparison paths in the recognition process.] 
A partir du point obtenu; on réitère la procédure jusqu'à ce que $X_{j}$ soit égal à $X_{N}$ ou bien $Y_{i}$ à $Y_{p}$.

La fonction coût optimale (soit $S$ le "score final ") est obtenue en sommant les fonctions coûts intermédiaires, c'est-à-dire les scores partiels $s_{k}$ :

$$
S=\sum_{k=1}^{K} s_{k}
$$

\section{$K:$ nombre total de pas}

L'avantage essentiel de la comparaison dynamique réside dans la normalisation temporelle qu'elle réalise, par la prise en compte automatique des répétitions ou des omissions dans les séquences d'échantillons (Fig. 6). Par contre, le choix du chemin optimal étant fait localement et non en considérant l'ensemble, il peut en résulter des erreurs de parcours et, par suite, un taux de dissemblance plus élevé qu'il ne devrait l'être.

Soient, par exemple, deux occurrences d'un même mot par le même locuteur. En principe, le chemin optimal devrait suivre d'assez près la diagonale principale du graphe de la figure 7. Par suite d'une dissimilitude locale importante dans la prononciation d'un phonème déterminé, le chemin trouvé peut s'en éloigner sensiblement et, de ce fait, le taux de dissemblance obtenu se trouver accru de façon excessive, ce qui risque de conduire ensuite à des conclusions erronées. Ceci est illustré sur la figure 7.

Les chiffres indiqués sont les distances $\mathrm{d}(i, j)$ entre un échantillon $Y_{i}$ du mot $M_{p}$ à reconnaître et un échantillon $X_{j}$ du mot $M_{1}$ du lexique. On a tracé, en trait plein, le chemin trouvé par l'algorithme de comparaison dynamique. Il lui correspond un score total $(S=16)$ supérieur à celui obtenu en suivant la

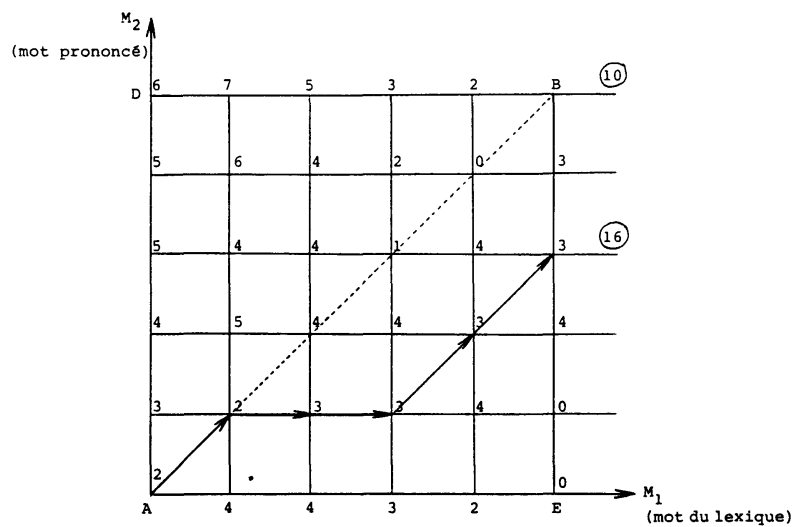

Fig. 7. - Exemple de calcul de scores pour deux occurrences d'un même mot prononcé par un seul locuteur.

[Typical score computations corresponding to two different occurrences of the same word said by one speaker.]

diagonale $(S=10)$. Il peut arriver que la comparaison avec un autre mot $\mathbf{M}_{2}$ du lexique conduise à un score inférieur à 16 , si bien que le processus de reconnaissance décidera que le mot prononcé ressemble davantage à $\mathbf{M}_{2}$ qu'à $\mathbf{M}_{1}$-conclusion qui est inexacte.

On peut pallier à cet inconvénient :

- soit en limitant arbitrairement les écarts permis par rapport à la diagonale ;

- soit en favorisant davantage encore la diagonale principale par pondération préférentielle des scores partiels à l'extrémité des chemins;

— soit en combinant les deux méthodes.

\section{Bibliographie sur la « comparaison dynamique »}

[1] KaufmanN, A. et Cruon, R., La programmation dynamique (Ed. par Dunod) 1965.

[2] Bellman, R., Dynamic programming (Ed. par Princeton University Press) 1957.

[3] LAURIÈRE, J. L., Eléments de programmation dynamique (Ed. par Gauthier-Villars) 1979.

[4] Sakoe, H. et Chiba, S., I.E.E.E. Trans. Acoust. Speech Signal Processing 26 (1978) 43.

[5] Myers, C. S., Rabiner, L. R., Rosenberg, A. E., I.E.E.E. Trans. Acoust. Speech Signal Processing 28 (1980) 622.

[6] Querre, M., Mercier, G., Gresser, J. Y., Note technique du C.N.E.T. Lannion CEI/CSI/44 (1974).
[7] Haton, J. P., Actes du 7th Int. Cong. on Cybernetics Namur (1973).

[8] White, G. M., Proc. of the I.E.E.E. Int. Conf. Acoust Speech Signal Processing (1978) 413.

[9] Myers, C. S. et Rabiner, L. R., I.E.E.E. Trans. Acoust. Speech Signal Processing 29 (1981).

[10] Rabiner, R. et Levinson, S. E., I.E.E.E. Trans. Commun. 29/5 (1981) 621.

[11] FoRney, G. D., Proc. I.E.E.E. 61 (1973) 268.

[12] LeA, W., Trends in Speech Recognition (Ed. par Prentice-Hall) 1980. 